\title{
Authentication with User Driven Web Application for Accessing Remote Resources
}

\author{
Yige Wang \\ The University of Texas at Austin \\ Austin, Texas \\ yige.wang@utexas.edu
}

\author{
Ruizhu Huang \\ Texas Advanced Computing Center \\ Austin, Texas \\ rhuang@tacc.utexas.edu
}

\author{
Weijia Xu \\ Texas Advanced Computing Center \\ Austin, Texas \\ xwj@tacc.utexas.edu
}

\begin{abstract}
With the emerging of data-driven analysis and data science, the needs of high-performance computing resources and cyberinfrastructure (CI) have been widely spread across almost all domain fields in academia. While CI providers have continued success with the infrastructure-as-a-service model (IaaS), there are increasing demands to offer more service models for diverse users and use cases. We have proposed a web application framework, which empowers users running their own analytic workflows as a web service that is accessible by other users authenticated with existing credentials. Through this framework, ad-hoc analysis routines can be described and wrapped in a workflow model that is shareable and re-useable. While the framework offers a new way for any user with valid credential to access and utilize remote resources, there are cases where more flexible authentication methods can further lower the access barrier of HPC resources. In this paper, we detail credential management of this framework and report our preliminary works on enabling temporary access to the remote resources including using dynamic generated credentials and social logins.
\end{abstract}

\section{CCS CONCEPTS}

- Security and privacy $\rightarrow$ Security services privacy; Access Control • Applied computing $\rightarrow$ Education

\section{KEYWORDS}

User driven web application, Cyberinfrastructure, authentication

Permission to make digital or hard copies of all or part of this work for personal or classroom use is granted without fee provided that copies are not made or distributed for profit or commercial advantage and that copies bear this notice and the full citation on the first page. Copyrights for components of this work owned by others than ACM must be honored. Abstracting with credit is permitted. To copy otherwise, or republish, to post on servers or to redistribute to lists, requires prior specific permission and/or a fee. Request permissions from Permissions@acm.org.

PEARC'18, July 22-26, 2018, Pittsburgh, PA, USA

(C) 2018 Association for Computing Machinery.

ACM ISBN 978-1-4503-6446-1/18/07 .. \$15.00

https://doi.org/10.1145/3219104.3229290
ACM Reference format:

Y. Wang, R. Huang, and W. Xu. 2018. Authentication with User Driven Web Application for Accessing Remote Resources. In Proceedings of ACM Practice and Experience in Advanced Research Computing Conference, Pittsburgh, PA, USA, fuly 2018 (PEARC '18), 7 pages. https://doi.org/10.1145/3219104.3229290

\section{INTRODUCTION}

Fueled by massive amounts of complex data produced by businesses, scientific applications, government agencies and social applications, data-driven analytics has the potential to help users gain new insights for decision making, scientific discovery, business insight, marketing potentials and more [1]. One of the key facets in realizing this promise has been the new techniques and tools for conquering extremely large datasets with the latest advances of computing infrastructures [2-4]. Across many domain science fields in academia, there is an increasing trend to move computational expensive analytics from standalone workstations to remote advanced computing resources such as a commercial cloud or cyberinfrastructure funded by various agencies and maintained in various high performance computing centers nationwide [5-7]. While centralized remote resources can provide potentially costeffective solutions, the complexity of infrastructure and current access models present challenges to users who are not used to remote computing environments. One exemplar case is to effectively support education on how to use those essential tools and computing resources that are important parts in preparing new data scientists and students in domain science fields [8].

To improve the CI resource accessibility for wide variety of use cases and domain fields, we propose a web application framework that allows users to login with remote authentication service providers using OAuth 2.0 protocol and dynamically supports various analysis pipelines by enabling users to set up their own interactive web application which interoperate with existing resources and services. The proposed framework follows model-view-controller design pattern. Common operations are abstracted as tasks. In addition to data model, each task also has a template for web application interface. Multiple tasks can be composed to a workflow through a simple configuration file in JSON format to describe operations required to accomplish a use case. The framework can then generate a web user interface based on the configuration file to allow users to run the analysis interactively on remote resources. Unlike existing tools for web portal and workflow management, the proposed framework includes pre-built support of provisioning resources, dynamic 
credential and access control, and self-contained web server that can run without requiring alleviated system privileges.

Among challenges of lowering the access barrier, enabling temporary access to remote resources is a practical requirement in some use cases but often ignored. One of such example is providing training sessions for participants who haven't used the resource before. In order to allow participants to participate in the hands-on activities, each participant must have access to the remote resources. There are two common solutions. One is to ask users to sign up for the account before or during the session. However, signing up new account often takes several steps and distracts the attention of participants away from following the training materials. Additionally, new account has to be added into some project allocation or reservation in order to allow user access. The other common practice is to provide user a prepared training account beforehand. Recipients of the accounts are often asked to sign their names and email addresses to acknowledge the receiving of the accounts for bookkeeping purposes. The temporary accounts need to be closed afterwards. Both practices are distractions to instructors and other participants of the training sessions. Another example where a temporary access becomes handy is that users may want to temporarily share their analysis process with others for collaboration purposes. Therefore, enabling temporary access, while should be avoided for long-term users, is needed in some practical use cases.

On the other hand, login through social media has become increasingly popular in recent years. Many websites allow potential users to sign up through their existing social media accounts such as through Facebook or Google+ accounts [9, 10]. The practice not only provides convenience for users but also helps service providers attract new potential users with additional trustworthiness.

Here, we explore alternative ways to authenticate a user including generating temporary account and utilizing user's existing social media account. The approach presented here is different from existing support models of using cloud and highperformance computing resources with following contribution highlights:

- On-demand user credential creation and management.

- Authentication through other service providers.

Although our motivation and preliminary work is to enable our user driven web application to be used as a virtual educational tool to support trainings, the model presented here may also be adopted for existing service providers to expand the current authentication methods.

The rest of the paper is organized as following. In Section 2, we review several typical usage models of existing cyberinfrastructure and how our proposed approach differs from those existing models in detail. In Section 3, we give an introduction of our user driven web application framework. Section 4 describes the design and implementation of the proposed authentication framework. In Section 5, we demonstrate features of our preliminary implementations. We conclude and briefly discuss ongoing works in Section 6.

\section{BACKGROUND AND RELATED WORK}

\subsection{Existing Cyberinfrastructure Service Models}

In academia, CI has been increasingly used in open science research and enabled break-through discovery in many domains. CI providers now face challenges to meet growing demands. We identify three utilization models that are most common within CI communities for open science.

fob Submission with Secure Shell Connection This mode requires user connecting to the $\mathrm{CI}$, typically through a dedicated login node on remote source, using a client application via Secure Shell Connection (SSH) protocol. All interaction between user, CI, and the application is through the command line interface (CLI).

Remote Software Session In this scenario, a user can connect to software service running at remote CI resources using client software installed locally. The user may have to manually start the software service at remote resource before making the connection. Although analysis environment and remote visualization can be supported, both number of choices and potential accessibility are still limited. The interoperability with additional resources and software tools and shareability among users are also limited.

Web Portal and Gateway A web portal (also known as gateway, user portal, community portal) is a website that is dedicated to users from a particular domain or sharing common needs on specific resources. The website has its own credential management that allows users to log on. A web portal often contains a set of features and tools that can simplify the process of using CI for a set of common use cases for its users.

The proposed application framework is compatible with the three service models described above and offers feature commentary to the existing services. With IaaS model, users can start a customized web application easily with improved usability and interactivity. CI providers have the flexibility to deploy the proposed framework with specific workflow defined as a software service for a targeted user community. The framework can also be deployed and made available as part of platform to support dynamically created workflows.

\subsection{Scientific Workflow Management Tools}

The framework also relates to widely available workflow management systems from open source community and industry [11-14]. The framework presented here shares similarity with existing scientific workflow tools in customizability and reconfigurability by users. However, a key difference is that the proposed framework is designed to run as a standalone web application. Therefore, it doesn't rely on server tools or software installation at remote resources. Hence, the proposed framework can be easily run on different remote resources and even with the potential to run in a heterogonous resources environment. Additionally, a key component of the proposed framework is the credential management that enables fine-grained access control and the ability to generate new credential dynamically. This 
component guarantees not only the defined workflow can be shared as file but also the analytic instance can be accessed by multiple users.

\subsection{OAuth Authentication}

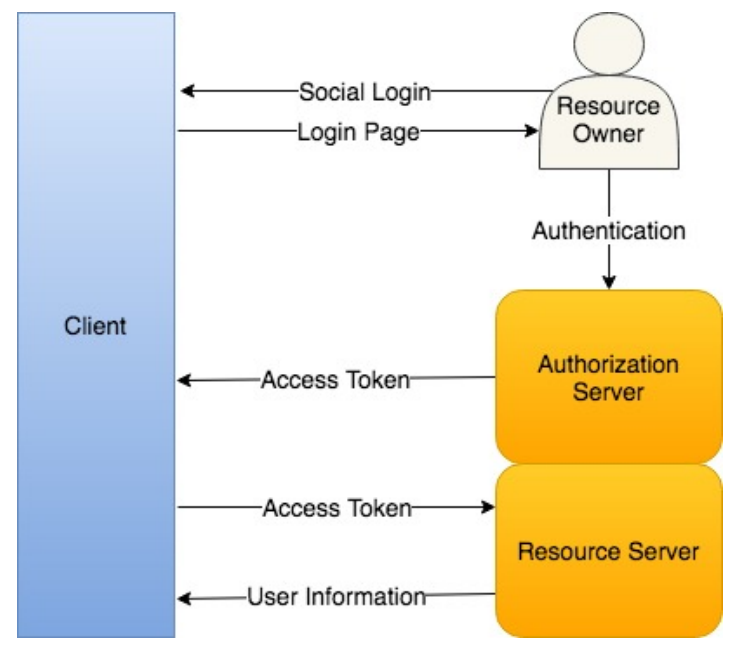

Figure 1: OAuth 2.0 protocol flow.

Millions of websites have implanted Social Logins through social networks because of its simplicity and convenience [15].

One of the most popular standard is the OAuth 2.0 authorization protocol, shown in Fig. 1. There are four roles defined by OAuth 2.0. Resource owner is the user who grants an application limited access to user's information. Social platform, as the resource server, holds this protected information, which is only granted to a request with valid access token. Authorization server, usually the same server as resource server, verifies the user's identity and issues access token to client, the application that requests this information [16]. With Web-based Single SignOn (WSSO), the framework can authenticate users based on their credentials from social networks including Facebook, Google+, and more $[9,10]$. This allows users to sign in easily and reduces the access barrier to advanced computing resources effectively.

\section{ARCHITECTURE DESIGN AND DATA MODEL}

An overview of the system architecture of the proposed framework and startup workflow is shown in Fig. 2 [17]. The web application can be started by resource providers and made available as a service directly to application users (SaaS). The proposed framework also enables application users to start the web application. In this case, application owner and application user are the same individual, and the resources providers just provide their infrastructure as a service (IaaS).

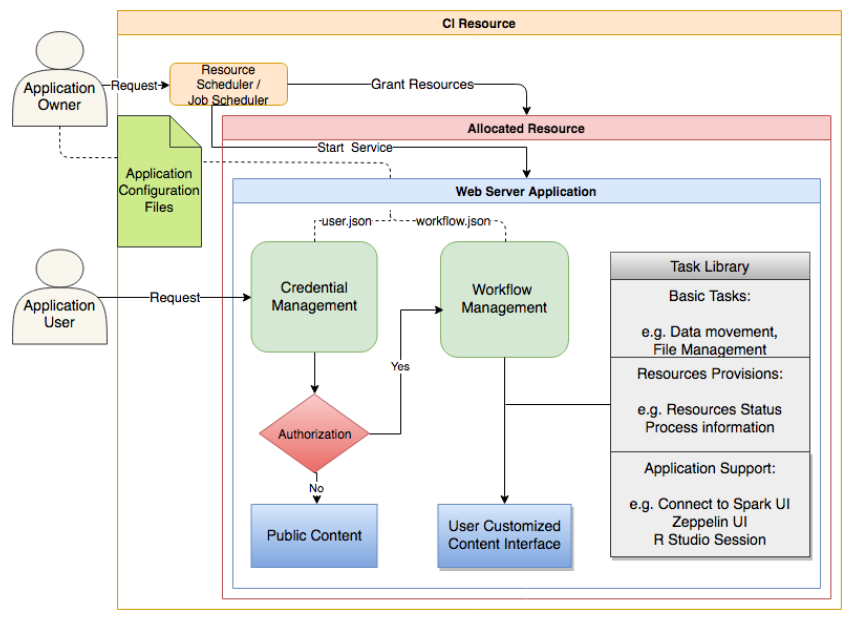

Figure 2: Overview of framework architecture and access workflow.

There are four key components of the proposed web application framework: credential management, workflow management, pre-defined task libraries, and application configuration files. The application configuration files are central in the proposed web framework. There are several types of configuration files for application parameters, user configuration, and workflow configuration. Each of the configuration files is in JSON format with a list of predefined fields and values. Many of the features and contents of the web application can be dynamically customized through configuration files. For example, the credential management component can use configuration files to initialize application user credentials. The workflow management component can generate customized workflow composed by tasks, which are pre-defined in task libraries, specified in configuration files.

\section{CREDENTIAL MANAGEMENT DESIGN}

\subsection{Design Overview}

The credential management provides access control support to other services available through the web application. There are two key modules, user service module and authentication module. The user service module supports common user management functions such as signing in, signing up, and generating new users. The authentication module includes authentication service interface and secure credential repository management. Fig. 3 shows an access workflow of credential management component. 


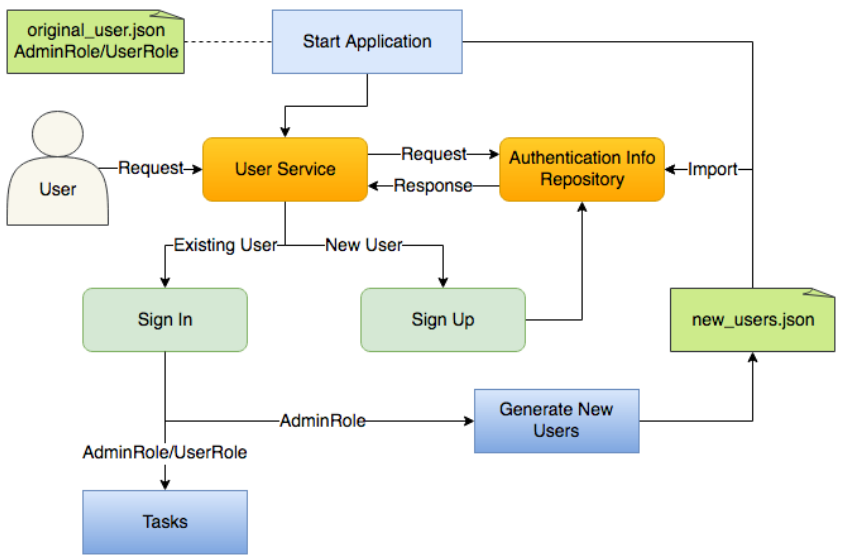

Figure 3: Overview of credential management component.

\subsection{User Management}

Each user object includes several basic fields including name, email, password, and role. Users can be defined using configuration files in JSON format and available upon start of the web application. Fig. 4 shows an example of a basic user object and corresponding JSON data.

\begin{tabular}{|l|}
\hline \multicolumn{1}{|c|}{ User } \\
\hline + First Name \\
+ Last Name \\
+ Email \\
+ Password \\
+ Role \\
\hline
\end{tabular}

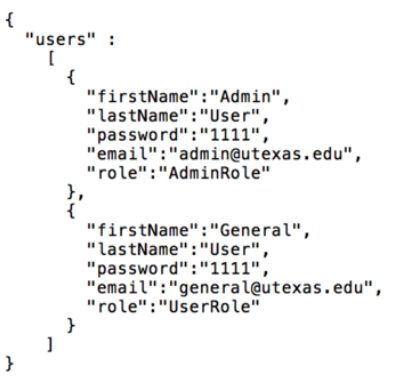

\section{Figure 4: Native user definition and exemplar user configuration files.}

There are two types of user accounts, admin accounts and user accounts. An admin user can be specified through the configuration file by the "role" field. By default, the user starts the web application will be designated as admin user. The admin accounts have additional privilege such as setting up applications, updating processing workflow, and creating new users.

In the use case of training sessions, the instructor, an admin user, can generate new regular users dynamically for students to use. Each user account has a random sequence of characters as password and user role. Shown in Fig.5, the information of the new users is saved as a JSON object to a file. The file is then used to save the user objects to the repository as shown in Fig. 3 such that students can use these users directly to sign in.

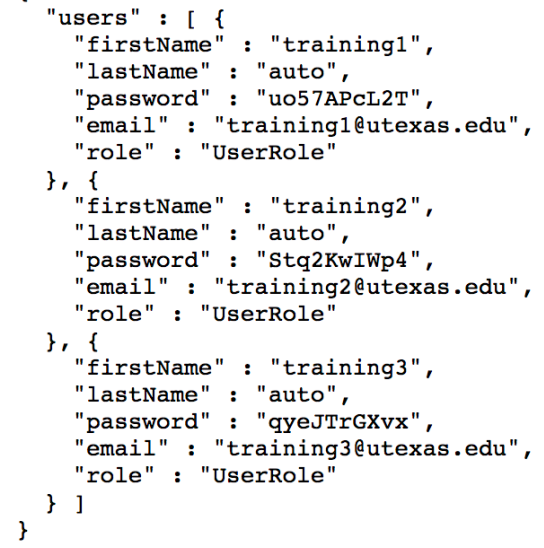

Figure 5: Exemplar admin-generated users.

Besides using the authentication service to track users, the framework saves all dynamic generated users to a file with limited access permission. To guarantee no user account is duplicated, the framework uses user emails as a unique identifier and checks both service module and file for user emails.

\subsection{Using External Credential Services}

In addition, we design our user management such that each user object can be linked with additional service provider accounts or social credentials. As shown in Fig. 6, the framework supports user login from two authentication providers, TACC and Facebook.

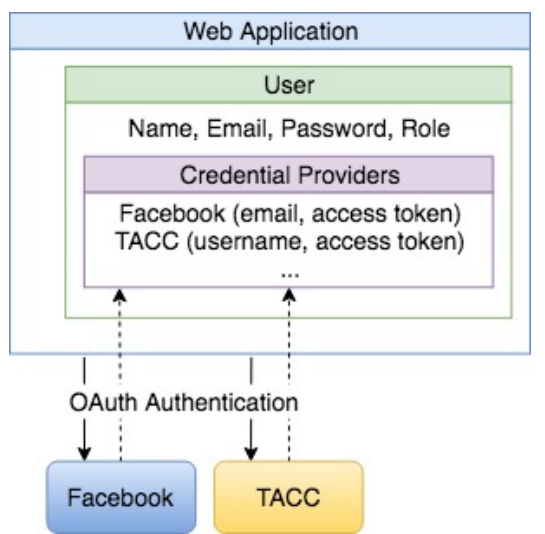

Figure 6: User linked with multiple credentials.

Similar to other OAuth 2.0 supported providers, an access token is provided to the framework when a user signs in with existing TACC or Facebook account [18, 9]. With the access token, user information such as name and email are retrieved. In the case when a student signs in, this information is added to the provider specific field of one of the user objects generated by the instructor. TACC account is necessary to access computing resources at TACC. For users without such account, the 
instructor needs to prepare training accounts ahead of time. Instead of marking the account with names and email addresses, participants can sign in with Facebook credentials. Once the identity is verified, a regular user account for the framework is automatically created and linked with one of the training accounts prepared. In this case, the Facebook account is used to authenticate the user's identity, and the training account is used for user to access remote resources.

\section{PRELIMINARY RESULTS}

\subsection{Implementation and Testing Environment}

We have implemented the proposed framework using Play Web Framework (version 2.6) [19]. Play framework is a Java and Scala based modern web development framework. It has several features especially suited for on-demand web application. It supports reactive web programming so that the content of web front can be dynamically updated along with the backend analysis progress. It includes a built-in lightweight web server, Jetty. This can greatly simplify the web UI deployment since it does not require any other additional system software or libraries other than Java. Play uses a template engine, Twirl, to generate web content. With template engine system, each web page is a result of a function call. Therefore, the content of web page is configurable through configuration files and the web page template files. Since the web page is in fact a Scala function, it can directly manipulate in memory data object with complex structure.

The authentication and authorization services are implemented using Silhouette authentication library (version 5.0). Silhouette supports several authentication methods, including OAuth1, OAuth2, OpenID, CAS, Credentials, Basic Authentication, and custom authentication schemes [20].

For development and testing purpose, we used Wrangler cluster at Texas Advanced Computing Center as remote computing resource test-bed. Wrangler cluster has been designed to support the data storage and sharing capacities of the system to enable data research [21]. It supports dynamically Hadoop cluster provisioning and a number of common data analysis and machine learning software tools and libraries.

\subsection{User Login Interface}

Fig. 7 shows the initial sign in interface presented to a user once the web application has started on the remote resources. In our preliminary implementation, we provide three credential options, the built-in credentials (via "Sign in" button), existing TACC user credentials (via "Sign in with TACC" button), and existing Facebook account (via the Facebook button). The builtin credentials are credentials specified in the configuration files before the application starts. All built-in credentials will be mapped to the credential used to start the web application when using the remote resources. The TACC credentials will be authenticated through the Agave web interface maintained at TACC [22]. Users authenticated via TACC to the web application will use their own credentials when accessing the remote resources at TACC.

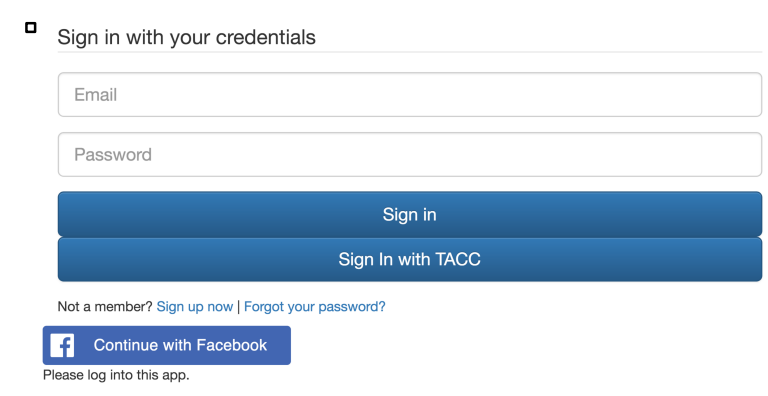

Figure 7: Login interface of the dynamically started user driven web application.

The Facebook credentials are supported through the credential services provided by Facebook. Fig. 8 shows the subsequent interface for authentication provided by Facebook service after user click the Facebook login button shown in Fig. 7 [23]. Users logged on via Facebook credentials can be assigned with a temporary credential from a predefined pool for using the remote resources.

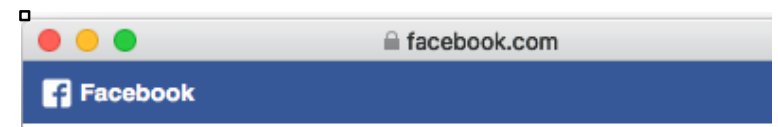

Log in to use your Facebook account with Idols.

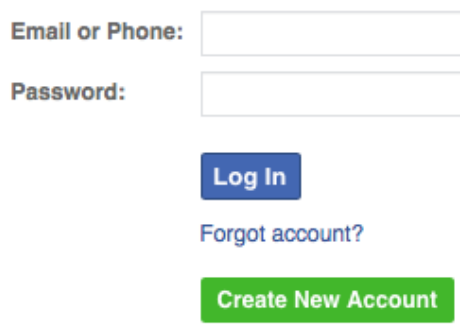

Figure 8: Pop-up window of using Facebook sign in option.

The application owner can configure available options and specific list of built-in user accounts through configuration file to tailor to specific need for different use case. Only built-in user accounts could be assigned with "admin" role with additional application access at run time.

\subsection{Support of Different User Roles}

Fig. 9 shows a screenshot of an admin user log on to an exemplar web application. As designated as "Admin", one of the features is to generate additional built-in user credentials dynamically. This feature is designed to meet the needs of granting temporary access on-demand such as during a training session. 


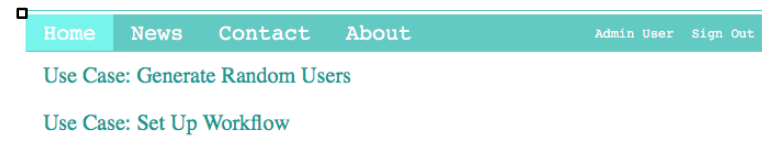

Figure 9: Screenshot of initial web interface presented to user with Admin role.

The admin user will also have more dynamic controls of the contents of the web application as shown in Fig. 10. In the "Workflow Management" module, an admin user can dynamically update and configure the workflow defined by the web application. This change will subsequently be reflected to all other users' logon to the web application as well.

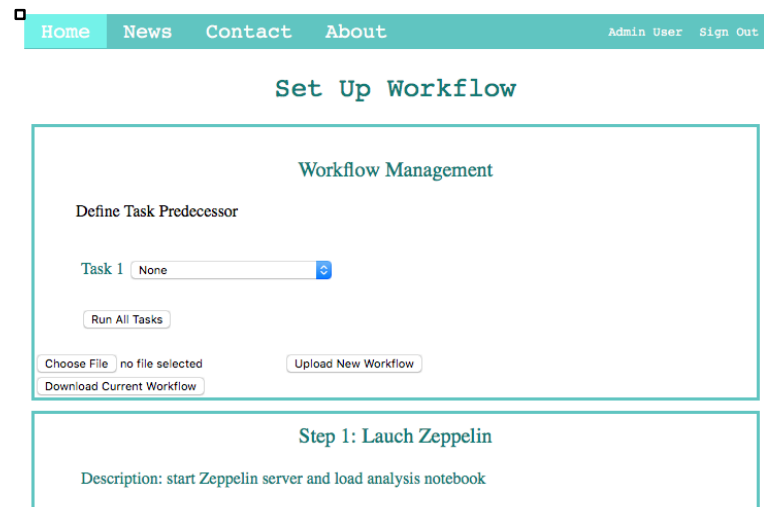

Figure 10: Workflow management for user designated as admin.

For comparison, we also show the corresponding screenshots for user log on as regular user using TACC credential service in Fig. 11. Fig. 11 shows fewer available options in the initial interface (top) and in the workflow management interface (bottom).

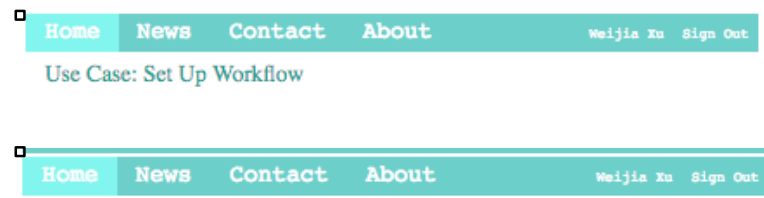

Set Up Workflow

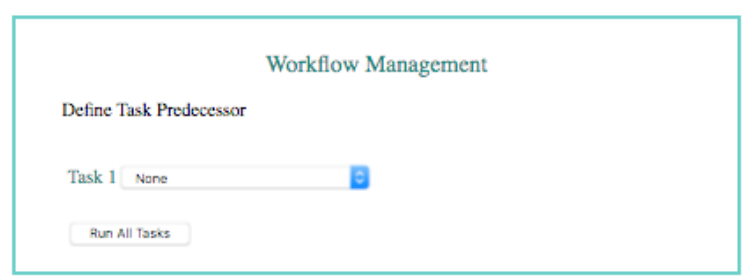

Figure 11: Screenshots for regular user's initial interface page (top) and workflow management page (bottom).

\section{SUMMARIES AND ONGOING WORK}

In this paper, we have presented credential management of our user driven web application framework. A key motivation of this project is to use the framework to enhance the accessibility of large cyberinfrastructure to users from diverse domain fields. The framework enables cyberinfrastructure users to login through authentication providers and setup their own web services easily. Our goal is to provide convenience for both resource providers and users when using the resources while maintaining the security and integrity of the remote resources. Towards that end, all credentials of the web application are only valid for limited duration as the web application runs on the remote resources. When the web application owner shutdown the running session, users without existing accounts will lose their access to the remote resources. The web application uses existing external credential services without the need to save user's external credential as well.

Our ongoing work includes configuring more authentication providers to allow flexible use of multiple identities. For users with regular TACC credentials, their Facebook credentials can be linked to their user accounts to allow easier login. Accessing the web application from either credential is treated as one user.

A specific aim of the project is to use the framework as a foundation for training and education activities of cloud computing. Educators and instructors can easily compose, stage and publish interactive web session on remote resources. Students can easily access and participate. The framework can be used as a way to set up cloud based virtual laboratory for training and education on advanced computing technology.

\section{ACKNOWLEDGMENTS}

This work has been supported through funding from National Science Foundation (Award \# 1726816). Software testing and demonstrations have been supported with Wrangler, an NSF funded cyberinfrastructure resource (Award \# 1341711).

\section{REFERENCES}

[1] Gordon Bell, "Foreword," in The Fourth Paradigm: Data-Intensive Scientific Discovery, Tony Hey, Stewart Tansley, and Kristin Michele Tolle, Eds. Redmond, MA: Microsoft Research, 2009, pp. Xi-XV.

[2] Tom White, Hadoop: The definitive guide.: O'Reilly Media, 2012

[3] Weijia Xu, Ruizhu Huang, Hui Zhang, David Walling, and Yaakoub ElKhamra, "Empowering R with High Performance Computing Resources for Big Data Analytics information," in Conquering Big Data with High Performance Computing, R. Arora, Ed.: Springer., 2016, pp. 191-218.

[4] Matei Zaharia et al., "Resilient distributed datasets: A fault-tolerant abstraction for in-memory cluster computing," in Proceedings of the 9th USENIX conference on Networked Systems Design and Implementation, 2012, p. 2 .

[5] Merchant, Nirav, et al., "The iPlant Collaborative: Cyberinfrastructure for Enabling Data to Discovery for the Life Sciences," PLOS Biology, 2016.

[6] Stephen A. Goff et al., "The iPlant collaborative: cyberinfrastructure for plant biology," Frontiers in plant science, no. 2, p. 34, 2011.

[7] E.M. Rathje et al., "DesignSafe: new cyberinfrastructure for natural hazards engineering.," Natural Hazards Review, vol. 18, no. 3, 2017.

[8] Avita Katal and Mohammad Wazid and R. H. Goudar, "Big data: issues, challenges, tools and good practices," in In Contemporary Computing (IC3), 2013 Sixth International Conference on., 2013, pp. 404-409.

[9] Facebook. [Online]. http://www.facebook.com/

[10] Google+. [Online]. https://plus.google.com/

[11] Diimitrios Georgakopoulos and Mark Hornick and Amit Sheth, "'An overview of workflow management: From process modeling to workflow automation 
infrastructure," Distributed and parallel Databases, vol. 3, no. 2, pp. 119-153, 1995.

[12] Suresh Marru et al., "Apache airavata: a framework for distributed applications and computational workflows," in In Proceedings of the 2011 ACM workshop on Gateway computing environments, 2011, pp. 21-28.

[13] Ilkay Altintas, Chad Berkley, Efrat Jaeger, Matthew Jones, and Bertram Ludascher and Steve Mock, "Kepler: an extensible system for design and execution of scientific workflows," in 16th Scientific and Statistical Database Management 2004, 2004, pp. 423-424.

[14] Malcolm Atkinson, Sandra Gesing, Johan Montagnat, and Ian Taylor, "Scientific workflows: Past, present and future," Future Generation Computer Systems, no. 75, pp. 216-227, 2017.

[15] Gafni, R., \& Nissim, D, "To social login or not login? - Exploring factors affecting the decision, " in Issues in Informing Science and Information Technology, vol. 11, pp. 57-72, 2014.

[16] Hardt, D., Ed., "The OAuth 2.0 Authorization Framework", RFC 6749, DOI 10.17487/RFC6749, October 2012, <https://www.rfc-editor.org/info/rfc6749>

[17] Weijia Xu, Ruizhu Huang and Yige Wang, Enabling User Driven Web Applications on Remote Computing Resource, in proceedings of IEEE Service 2018, July 2-6, San Francisco, CA, 2018

[18] Texas Advanced Computing Center. [Online]. https://portal.tacc.utexas.edu/

[19] Play Development Team. Play Web Framework. [Online]. https://www.playframework.com/

[20] Silhouette. Silhouette authentication library. [Online]. https://www.silhouette.rocks/

[21] Christopher, Jordan, David Walling, Weijia Xu, Stephen A. Mock, Niall Gaffney, and Dan Stanzione "Wrangler's user environment: A software framework for management of data-intensive computing system," in 2015 IEEE International Conference on Big Data, 2015, pp. 2479-2486.

[22] Agave API. [Online]. https://agaveapi.co/

[23] Facebook Login. [Online]. https://developers.facebook.com/docs/facebook$\operatorname{login} / w e b /$ 